\title{
Acute Coronary Syndrome: Insights Based on Intravascular Imaging
}

\author{
Shigenobu Inami, Masamichi Takano, Kyoichi Mizuno
}

\begin{abstract}
Intravascular imaging devices can offer information about vessels that cannot be depicted by angiography. Intravascular ultrasound (IVUS) can image the trilaminar structure of vessel walls and not only enable quantitative analysis, but also tissue characterization by radiofrequency data analysis. Coronary angioscopy provides full color images as direct visualization of the coronary lumen, and the images are applicable to the macroscopic diagnosis of intracoronary structures. Optical coherence tomography (OCT) is a novel optical imaging technology that provides cross-sectional tomographic images that yield detailed structural information superior to any other currently available modality. These intravascular imaging devices have played an important role both in the elucidation of the pathology of coronary artery disease and in the guidance of clinical treatment. Rupture of atherosclerotic plaque and the overlying thrombus is often observed in culprit lesions of acute coronary syndrome (ACS). In vivo, intravascular imaging devices can show detailed morphology of the culprit lesion that is associated with the clinical presentation and the prognosis. Ruptureprone plaques are called "vulnerable plaques." Identification of vulnerable plaques has been attempted over the years. Prospective studies have shown that thin cap fibroatheroma (TCFA), defined by different intravascular imaging devices, is related to future cardiac events. Furthermore, multiple vulnerable plaques are often observed in non-culprit arteries of ACS patients. Therefore, ACS is recognized to represent a pancoronary process of vulnerable plaque development. In addition, the multiplicity of vulnerable plaques was also demonstrated to be a predictor of ACS events. Some cardiac biomarkers, which are reported to be predictors of ACS events, are associated with the characterization of culprit and non-culprit arteries in ACS patients. On the other hand, the detailed findings obtained by intravascular imaging devices play an important role to prove the validity of noninvasive coronary artery studies. Near-infrared spectroscopy and coronary thermography can reveal plaque vulnerability by detecting inflammation and by quantifying the molecular composition of the plaque. The introduction of new technologies will increase our understanding of the pathophysiology and prevention of ACS.

KEY WORDS: intravascular ultrasound, angioscopy, optical coherence tomography, plaque disruption, atherosclerosis
\end{abstract}

\section{Introduction}

Traditional coronary angiography only portrays a silhouette of the vessel lumen. Intravascular imaging devices can clarify the structural and tissue characteristics of vessels that cannot be depicted by angiography, and they play an important role both in the elucidation of the pathology of coronary artery disease and in the guidance of clinical treatment. In this review, the current intravascular imaging modalities will be discussed with the emphasis on intravascular imaging findings in patients with ACS.

Division of Cardiology, Department of Internal Medicine, Nippon Medical School, 1-1-5 Sendagi, Bunkyo-ku, Tokyo 113-8602, Japan Received October 12, 2011; Accepted December 5, 2011

\section{Intravascular imaging devices}

Intravascular ultrasound (IVUS) can provide tomographic images of the coronary artery and enable measurement of the vascular diameter, lumen area, plaque area and arterial wall remodeling index. The remodeling index (RI) is generally calculated from the ratio of the external elastic membrane (EEM) areas at the culprit lesion to the EEM areas at the proximal reference site. Positive remodeling is generally defined as $\mathrm{RI}>1.05$; negative remodeling as $\mathrm{RI}<0.95$. The echogenicity of the plaque area reflects the atherosclerotic component. Low-and high-intensity plaques represent lipid and fibrous contents, respectively. Calcifications within plaques are identified by the presence of bright ultrasonic reflections with acoustic shadows. Furthermore, the IVUS radiofrequency data analysis was developed to clarify the classification of different types of plaque components. On the one hand, integrated 
backscatter IVUS (IB-IVUS) can be used to analyze radiofrequency signals by applying fast Fourier transformation of the frequency components of the backscattered signals by calculating the intensity of the signal measured in decibels $(\mathrm{dB})$. The first commercially available tissue characterization tool based on RF-signal analysis was Virtual Histology $^{\mathrm{TM}}$ (VH-IVUS Volcano Therapeutics, Rancho Cordova, CA, USA). VH-IVUS constructs tissue maps that classify plaques into four major components to which four color codes are assigned. The calcified, fibrous, fibro-lipidic, and necrotic core regions are labeled white, green, light green, and red, respectively (Fig. 1). Correlation between the in vivo IVUS radiofrequency data analysis and histopathology shows a high level of accuracy. ${ }^{1)}$ However, VHIVUS has limitations for the assessment of intramural thrombi. The current classification tree used for the VHIVUS analysis cannot differentiate the presence of an intramural thrombus due to difficulty differentiating the between the borderline of an intramural thrombus and a fibrous plaque. Therefore, VH-IVUS falsely identifies the intramural thrombus as a fibrous or fibrofatty lesion. Recently, another RF-based processing method, iMAP-IVUS (Boston Scientific, Santa Clara, CA, USA), has become commercially available for coronary plaque tissue characterization.

Coronary angioscopy provides high resolution (10$50 \mu \mathrm{m}), 3$-dimensional, and full color images as direct visualization of the coronary lumen, and the images are applicable to the macroscopic diagnosis of intracoronary structures including atherosclerotic plaque, thrombi, and neointimal tissue based on the surface color and morphology. Even plaque erosions without lipid core rupture can be detected. Dye-staining coronary angioscopy using Evans blue dye can also identify mural fibrin and damaged endothelial cells (Fig. 2). ${ }^{2-4)}$ This procedure involves the injection of warmed, colorless, transparent solutions into the coronary artery during angioscopic observation to remove blood from the viewing field. Presently, two types of angioscopic catheters are available; one is an occlusion type, and the other a maintenance type with regard to the coronary blood flow. The occlusion type (VecMova Neo Fibertec Co, Chiba, Japan) is $4.5 \mathrm{Fr}$ in diameter and of the rapid-exchange type. The image bundle consists of 3,000 optical fibers with a micro-lens at the distal tip, and can be advanced $7 \mathrm{~cm}$ in front of the delivery catheter along a 0.014 inch guide wire. An occlusion balloon is located at the distal tip of the delivery catheter, and the balloon is inflated manually for the image acquisition. The maintenance type (Fiber Catheter AS-003 Fib- ertec Co, Chiba, Japan) consists of 6,000 optical fibers and needs a microcatheter for the delivery of optical fibers and flushing with Ringer's solution. Before the use of either angioscopic system, the white balance, light power, and focus are adjusted to acquire appropriate images.

Optical coherence tomography (OCT) is a novel optical imaging technology that provides cross-sectional tomographic imaging, which yields detailed structural information superior to any other currently available modality. The image is formed by backscattering of light from different sites of the tissue with a $15-20 \mu \mathrm{m}$ axial resolution, which is about ten times that of IVUS. However, OCT is associated with difficulty with imaging of the trilaminar structure of vessel walls at coronary atherosclerotic lesions because the near-infrared light signals are attenuated.

The different components of the visualized atherosclerotic lesion can be identified by their distinctive borderlines and the intensity, uniformity and attenuation of the associated signal. Fibrous plaques consist of homogeneous, high back-scattering areas with indistinct borderlines. Lipid plaques have diffuse borders, but exhibit lower signal intensity with high attenuation. Calcifications within plaques are identified by the presence of well-delineated, low backscattering heterogeneous regions (Fig. 3). Previous postmortem studies have demonstrated the excellent sensitivity and specificity of distinguishing between plaque characteristics by OCT.5, 6) The fibrous cap thickness of the lipid plaque, as measured by OCT and histological assessment, also shows close correlation (Fig. 4). ${ }^{7}{ }^{8}$

Plaque disruption and thrombi, which are frequently found within the culprit lesions of patients with ACS, can be identified by OCT. Thrombi are identified by OCT, as are well-delineated masses with high signal intensity that are attached to the vessel wall and protrude into the lumen. OCT can even distinguish between a red thrombus (high-backscattering protrusions with signal-free shadowing) and a white thrombus (low-backscattering protrusions). ${ }^{9)}$ Tearney, et al. also reported methods that allow the quantification of macrophages within the fibrous caps of atherosclerotic plaques. ${ }^{10)}$ A previous investigation of patients with ACS demonstrated that, in comparison with coronary angioscopy, OCT showed a similar frequency of thrombi detection and a higher frequency of plaque rupture detection.

The first commercially-available OCT system was the M2 OCT Imaging System (LightLab Imaging, Inc., Westford, MA, USA). The M2 OCT system uses a broadband light source that emits multiple wavelengths across a spectrum, and is also called time-domain OCT (TD-OCT). 


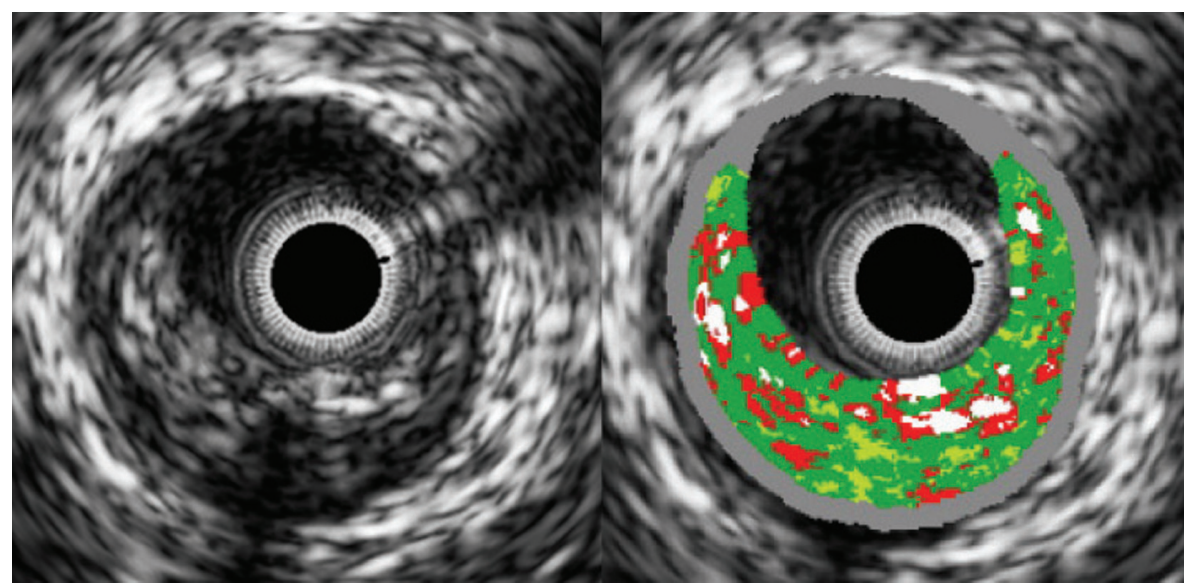

Fig. 1 The Virtual Histology ${ }^{\mathrm{TM}}$ system (VH-IVUS)

VH-IVUS constructs tissue maps that classify plaques into four major components, to which four color codes are assigned. Calcified, fibrous, fibro-lipidic, and necrotic core regions are labeled white, green, yellowish green, and red, respectively.

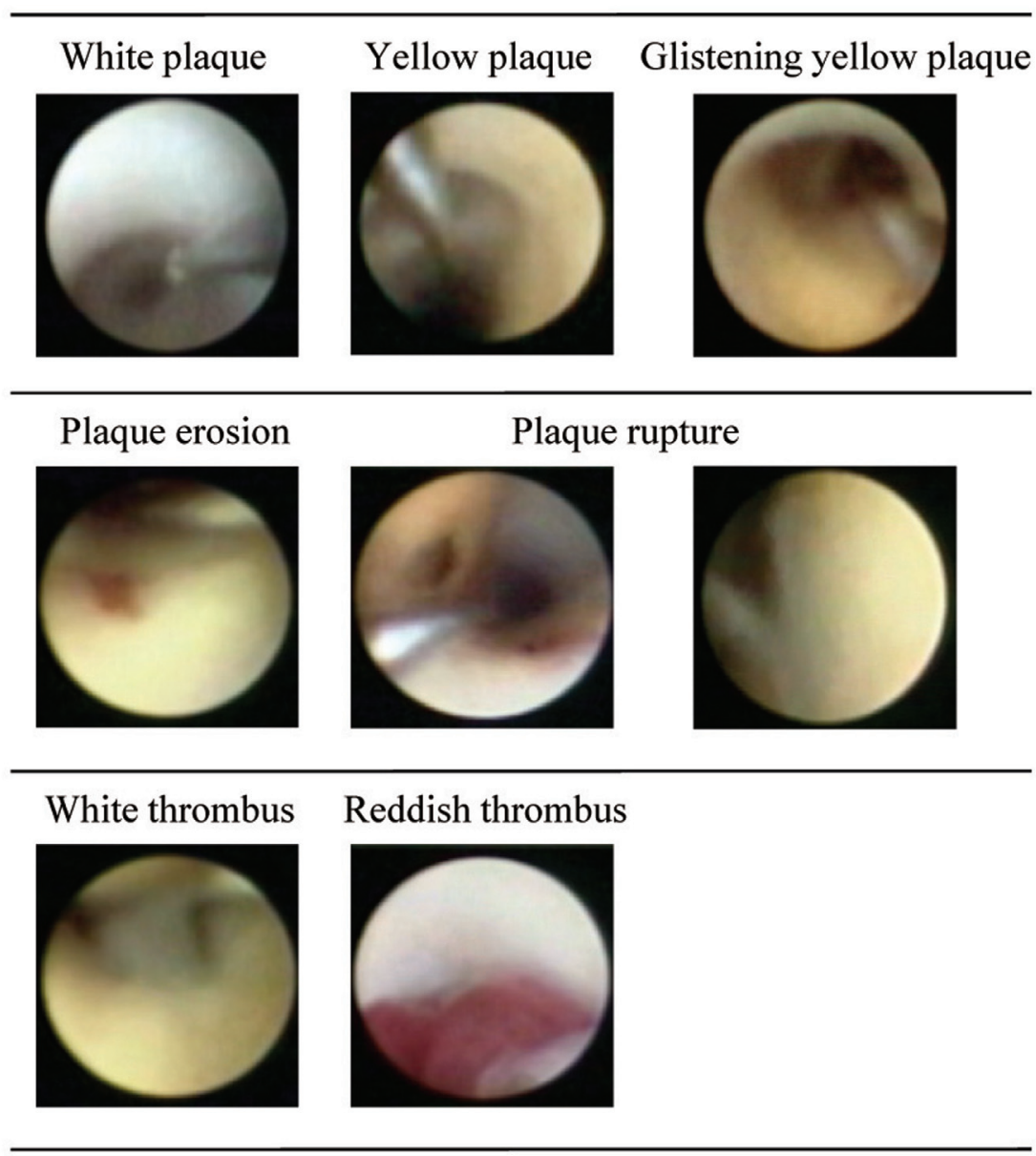

Damaged endothelial dyed by Evans blue

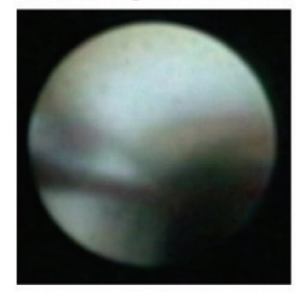

Fig. 2 Angioscopic images of coronary atherosclerotic lesions. 


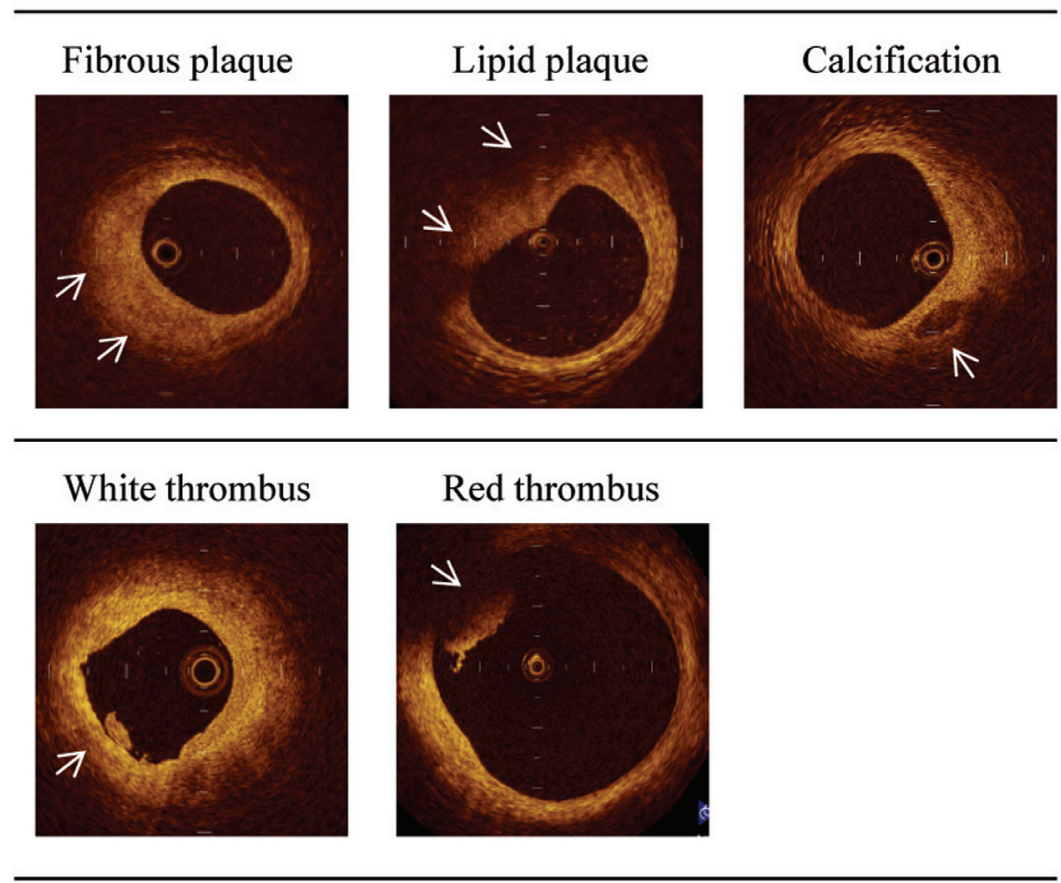

Fig. 3 Cross-sectional optical coherence tomography images of coronary atherosclerotic lesions.

Fibrous plaques are indicated by homogeneous high intensity areas, lipid plaques are indicated by irregular borders of a low signal intensity area with high attenuation. Calcification is noted by sharp borders, and heterogeneous low signal intensity areas with low attenuation. Red thrombi have a high intensity, and are associated with highbackscattering protrusions with high attenuation, while white thrombi have a high intensity with low-backscattering protrusions.

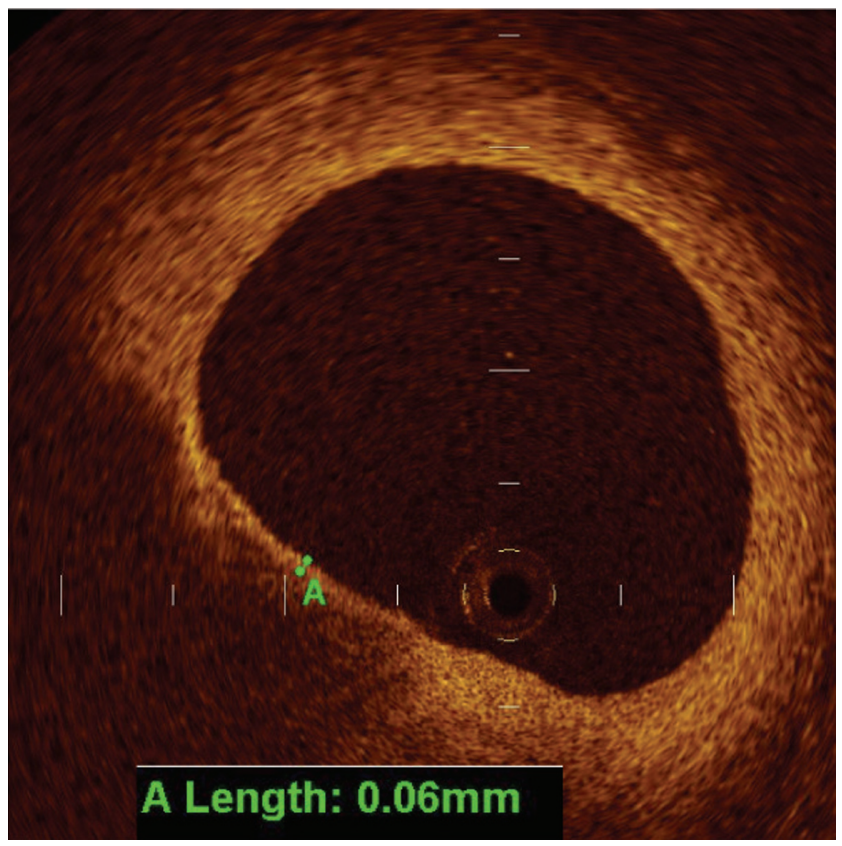

Fig. 4 Measurement of the fibrous cap thickness of a lipid plaque.

The fibrous cap thickness of the lipid plaque can be measured using OCT.
Since light-scattering occurs from red blood cells that may cause significant signal attenuation, complete removal of blood from the target vessel is necessary to acquire the images. TD-OCT generally requires an overthe-wire type catheter with an occlusion balloon (Helios ${ }^{\mathrm{TM}}$ Goodman, Co. Ltd., Nagoya, Japan) to obtain continuous long sectional images.

Recently, the C7-XR imaging system (LightLab Imaging, Inc., Westford, MA, USA) has become commercially available. The $\mathrm{C} 7$ system uses a novel swept frequency laser as the light source, and is also called frequency domain OCT (FD-OCT). The max frame rate is $100 \mathrm{f} / \mathrm{sec}$, and reaches speeds of five times that of previous generation OCT systems. The nominal pull-back speed of the C7 system is $20 \mathrm{~mm} / \mathrm{sec}$, which is ten times that of the M2 OCT systems. The C7 system has the potential to scan the target coronary vessel in its entirety within a few seconds. This feature permits the use of a single bolus flush to produce a blood-free environment, eliminates the need for balloon occlusion, and makes it possible to observe proximal lesions. Furthermore, the $\mathrm{C} 7$ system uses a monorail-type catheter, which allows for faster and 
Table 1 Characteristic intravascular findings of the culprit lesion in patients with acute coronary syndrome

Common findings

- Disrupted plaque and thrombus.

GS-IVUS

- Positive remodeling (greater in AMI than in UA).

- Noncalcific attenuated plaque.

VH-IVUS

- Large necrotic core.

- VH-TCFA, which is usually defined as a necrotic core $\geq 10 \%$ of the plaque area without overlying fibrous tissue in a plaque burden $\geq 40 \%$.

Angioscopy

- Grayish-white thrombi (more frequently in UA than AMI).

- Reddish thrombi (more frequently in AMI than UA).

- Plaque erosion without rupture (more frequently in small than large infarct size).

OCT

- OCT-TCFA, which was defined as a lesion with a fibrous cap thickness of $<65 \mu \mathrm{m}$.

- Ruptured cavity of plaque (larger in STEMI than NSTEACS).

- Ruptured plaque of which aperture open-wide against the direction of coronary flow (more frequently in STEMI than NSTEACS).

- Macrophage infiltration.

GS-IVUS: Gray scale-IVUS, VH-IVUS: Virtual Histology ${ }^{\mathrm{TM}}$-IVUS, VH-TCFA: VH-IVUS derived thin cap fibroatheroma, OCTTCFA: OCT derived thin cap fibroatheroma, AMI: acute myocardial infarction, UA: unstable angina, STEMI: ST elevation myocardial infarction, NSTEACS: non-ST elevation acute coronary syndrome

easier deployment into the coronary arteries. Terumo (Terumo Corporation, Tokyo, Japan) and Volcano (Volcano Corporation, Tokyo, Japan) are also developing new FD-OCT systems.

\section{ACS explored by intravascular imaging}

\section{Intravascular image findings of culprit lesions (Table 1)}

Pathology studies have shown plaque disruption and subsequent thrombus formation as the underlying ACS. Two-thirds of acute events are considered to be the result of rupture of a thin-cap fibroatheroma (TCFA), which is characterized histologically by the following features: a thin fibrous cap $(<65 \mu \mathrm{m})$, a large lipid pool, and activated inflammatory cells near or within the fibrous cap. In vivo intravascular imaging devices have clarified the relationship between the detailed morphology of culprit lesions and the clinical presentation of ACS.

When comparing ACS and stable coronary artery disease (SAP), IVUS studies have shown that positive remodeling (PR) is more frequently observed in the culprit lesions of ACS than in those of SAP.11) There are also differences in the echogenicity of culprit lesions between ACS and SAP patients. Noncalcified attenuated plaque identified by IVUS is often seen in patients with ACS, and is associated with no reflow during percutaneous coronary intervention. ${ }^{12)}$ Histopathology studies have shown choles- terol clefts, microcalcifications, or organized thrombi in the noncalcified attenuated plaques.13, 14) VH-IVUS makes it clear that the culprit tissue components vary by clinical presentation. The culprit lesions in patients with ACS have a larger necrotic core (NC) and are less fibrofatty compared with the target lesions in patients with SAP. VH-IVUS-derived TCFA (VH-TCFA), which is usually defined as a necrotic core of $\geq 10 \%$ of the plaque area without overlying fibrous tissue in a plaque burden of $\geq 40 \%$, more frequently exists in culprit lesions in patients with ACS than in those with SAP. ${ }^{15)}$ Noncalcified attenuated plaques are associated with a large VH-IVUS necrotic core. ${ }^{16)}$ MacNeil, et al. investigated the relationship between the detection of macrophages in the culprit lesion by OCT and the clinical presentation. A significantly greater macrophage density was found in unstable patients, both in fibrous and lipid-rich plaques. In culprit lesions, the surface macrophage infiltration was a stronger predictor of an unstable clinical presentation than the subsurface infiltration. ${ }^{17}$

When comparing unstable angina (UA) and AMI, an angioscopic study demonstrated that the culprit lesions of AMI tend to have a higher prevalence of thrombi, while the culprit lesions of AMI and UA patients have a similar yellow color intensity. ${ }^{18)}$ Furthermore, grayish-white thrombi are frequently observed in patients with UA, 
Table 2 Characteristic intravascular findings of the culprit lesion before acute coronary syndrome

IVUS

- Large eccentric plaque containing an echolucent zone in the shallow portion.

- Positive remodeling.

- VH-TCFA, which is usually defined as a necrotic core $\geq 10 \%$ of the plaque area without overlying fibrous tissue in a plaque burden $\geq 40 \%$.

- Large plaque burden $(\geq 70 \%)$ and a small minimal luminal area $\left(\leq 4 \mathrm{~mm}^{2}\right)$.

Angioscopy

- Yellow plaque.

- Glistening yellow plaque.

OCT

- OCT-TCFA, which was defined as a lesion with a fibrous cap thickness of $<65 \mu \mathrm{m}$.

VH-TCFA: Virtual Histology ${ }^{\mathrm{TM}}$ IVUS derived thin cap fibroatheroma, OCT-TCFA: OCT derived thin cap fibroatheroma

while reddish thrombi are frequently observed in patients with AMI.19) These angioscopic presentations may explain why thrombolytic treatment using tPA is less effective in patients with UA. According to the IVUS findings, the degree of PR is greater in AMI than in UA. ${ }^{11}$

When comparing ST elevation myocardial infarction (STEMI) and non-ST elevation acute coronary syndrome (NSTEACS), an OCT study demonstrated that plaque rupture, red thrombus, and TCFA occur more frequently in the culprit lesion of STEMI than in that of NSTEACS patients. Although the lumen area at the site of plaque rupture was similar in the two groups, the area of the ruptured cavity was larger in the STEMI than the NSTEACS patients. A ruptured plaque that is widely open against the direction of coronary flow is more often seen in STEMI than in NSTEACS patients. ${ }^{20)}$ Disrupted plaque is a common angioscopic finding in the culprit lesions of acute myocardial infarction (AMI). The infarct size is smaller in patients who have plaque erosion than plaque rupture in the culprit lesion.21)

2. Intravascular image findings of culprit lesions before ACS (Table 2)

It is difficult to identify vulnerable plaques angiographically, since atherosclerotic plaques that lead to ACS often occur at sites of mild coronary artery stenosis. The identification of vulnerable plaques before ACS using intravascular imaging has been attempted over the past few years.

In a 12-month prospective follow-up study, ACS occurred more frequently in patients with yellow plaques than in those with white plaques. ACS occurred particularly frequently in patients with glistening yellow plaques, and were detected within a short time. ${ }^{22)}$ Other studies have confirmed that the intensity of the yellow color of plaques as determined by coronary angioscopy was strongly related to the prevalence of thrombosis on the plaque. ${ }^{23)}$ The thickness of the fibrous cap measured by OCT is proportional to the intensity of the yellow color. ${ }^{7.8)}$ The fibrous caps of glistening yellow plaques are very thin, and it is anticipated that such thin caps will have a high tendency for disruption. These findings have established that yellow plaques are TCFA and that they also are vulnerable plaques that will likely lead to the development of ACS in the future. Since the intensity of the yellow color may be a marker of plaque vulnerability, quantitative colorimetry is applied to objectively assess the intensity of the yellow color. ${ }^{24)}$

One hundred and six patients with coronary atherosclerotic lesions identified by IVUS were prospectively followed. During the follow-up period of $21.8 \pm 6.4$ months, 12 patients had an acute coronary event. The preexisting plaques that led to the acute events had been noted to be large eccentric plaques containing an echolucent zone in the shallow portion. The lumen area was preserved at the time of the initial study due to positive remodeling. ${ }^{25)}$ The PROSPECT trial (a prospective natural-history study of coronary atherosclerosis) enrolled 697 patients with acute coronary syndromes who underwent three-vessel coronary angiography, and gray-scale and radiofrequency intravascular ultrasonographic imaging after percutaneous coronary intervention. The median follow-up period was 3.4 years. The 3-year cumulative event rate judged to be related to nonculprit lesions was $11.6 \%$ (104 lesions in 74 patients). Major nonculprit-lesion-related adverse cardiovascular events arose most frequently from thincap fibroatheromas with a large plaque burden $(\geq 70 \%)$ and a small minimal luminal area $\left(\leq 4 \mathrm{~mm}^{2}\right)$, or with some combination of these characteristics. In contrast, follow-up events rarely originated from nonfibroatheromas, regardless of the lesion severity. ${ }^{26)}$ On the other hand, positive 
Table 3 Characteristic intravascular findings of a nonculprit lesion in patients with acute coronary syndrome

IVUS

- Large necrotic core.

- VH-TCFA, which is usually defined as necrotic core $\geq 10 \%$ of the plaque area without overlying fibrous tissue in a plaque burden $\geq 40 \%$.

Angioscopy

- Intensive yellow plaque.

- Disrupted yellow plaque (more frequently in CKE-ACS than NCKE-ACS).

OCT

- OCT-TCFA, which was defined as a lesion with a fibrous cap thickness of $<65 \mu$ m.

- Elevated macrophage density.

VH-TCFA: Virtual Histology ${ }^{\text {TM }}$ IVUS derived thin cap fibroatheroma, OCT-TCFA: OCT derived thin cap fibroatheroma

remodeling showed no consistent relationship to the necrotic core. There are conflicting data suggesting that lesions with positive remodeling have a smaller percentage area of the necrotic core at the MLD site compared with intermediate/negative remodeling lesions, while the correlation between the lipid core and remodeling was reported to show a positive linear relationship. ${ }^{27)}$

3. Intravascular image findings of non-culprit lesions (Table 3)

Autopsy studies have suggested that AMI represents a pan-coronary process of vulnerable plaque development. According to the angioscopic examination of patients with ischemic heart disease, the yellow plaques were diffusely distributed in the right coronary artery but more prominently in the mid-segments of the left anterior descending coronary artery and the left circumflex coronary artery. Intensive yellow plaques and disrupted yellow plaques are more frequently detected in nonculprit segments of ACS than in non-ACS patients. Furthermore, a greater number of intensive yellow plaques and a higher prevalence of thrombosis were detected in Creatine Kinase Elevation-ACS patients compared to Non Creatine Kinase Elevation-ACS patients. ${ }^{28)}$

A 3-vessel IVUS study also demonstrated that ruptured plaque is frequently found somewhere else than in the culprit lesion in many patients with ACS. ${ }^{29}$ The plaque component in non-culprit vessels is associated with the clinical presentation. The percentage of necrotic core is larger in non-culprit vessels of ACS than in those of SAP patients. Conversely, stable patients show more fibrotic content. ${ }^{30}$ ) The frequency of VH-TCFA is higher in culprit and nonculprit lesions in patients with ACS than in those with SAP. Most of the VH-TCFA is located in the proximal artery. ${ }^{31,32)}$

Non-culprit segments have also been investigated by
OCT. In nonculprit segments, the fibrous cap thickness was significantly thinner in AMI than in SAP patients. The frequency of OCT-derived TCFA, which was defined as a lesion with a fibrous cap thickness of $<65 \mu \mathrm{m}$, was greater in AMI patients. ${ }^{33)}$ A significantly greater macrophage density detected by OCT was found in unstable patients, both for fibrous and lipid-rich plaques. The macrophage density of non-culprit lesions correlated with that of the culprit lesions. ${ }^{21}$

It is suggested that the morphology of non-culprit arteries has prognostic implications. There is the investigation of long-term clinical outcomes of 183 patients (79 with SAP and 104 with ACS) who underwent pre-intervention 3-vessel IVUS and single-vessel stent implantation. ${ }^{34)}$ In this study, vulnerable plaques were defined as plaques containing the following unstable features: rupture, lipid core, dissection, or thrombus. The multiplicity of vulnerable plaques in the non-target vessels was the only independent predictor of long-term critical events. Also on angioscopic examination, the multiplicity of yellow plaques was the only predictor of ACS events. ${ }^{35}$

\section{Biomarkers reflecting intravascular findings}

Intravascular imaging devices have been used to investigate whether serum biomarkers reflect the morphology and characteristics of coronary vessels. An elevated troponin $\mathrm{T}$ level is one of the predictors of an adverse outcome in NSTE-ACS patients. On angioscopic examination, elevated troponin $\mathrm{T}$ levels were associated with the presence of thrombi and yellow plaque at the culprit lesions of NSTE-ACS. Troponin T was considered positive when the level was $\geq 0.01 \mathrm{ng} / \mathrm{ml}$, and had a high specificity for detecting intracoronary thrombi. ${ }^{36,37)}$

C-reactive protein (CRP) is recognized as a strong risk predictor of cardiovascular events, and tissue factor plays 
a central role in thrombus formation of advanced atherosclerotic plaques. In coronary atherectomy specimens, complex plaques revealed a higher intimal CRP and tissue factor expression than white/yellow plaques detected by coronary angioscopy. The expression levels of CRP and tissue factor were markedly increased in plaques derived from patients with ACS compared with those from SAP patients. ${ }^{38)}$ The plasma CRP level is reported to have a positive correlation with the number of plaque ruptures in patients with AMI.39) In addition, a VH-IVUS study clarified that increased plasma CRP and hypoadiponectinemia are related to an elevated necrotic core ratio both in culprit and nonculprit lesions in patients with ACS.40) On OCT examination, there were inverse linear correlations between the fibrous cap thickness and plasma levels of inflammatory factors including CRP, IL-18, tumor necrosis factor alpha and the peripheral white blood cell count. ${ }^{41)}$

Matrix metalloproteinases (MMPs) are important for the resorption of extracellular matrices and may degrade the fibrous cap of an atherosclerotic plaque, thus contributing to coronary plaque rupture. An IVUS study demonstrated that there were high levels of MMP-9 in ACS patients, and that these were related to the presence of plaque rupture of the culprit lesion. ${ }^{42}$

\section{Intravascular findings during percutaneous coronary intervention}

In a comparison of the coronary arterial findings by IVUS in patients with "transient no-reflow" versus "reflow" during percutaneous coronary intervention for ACS, the amount of plaque burden in the culprit lesion was greater in the transient no-reflow group than in the reflow group. The rate of thrombus formation was also higher in the transient no-reflow group than in the reflow group. ${ }^{43)}$ On OCT examination, TCFA was more frequently observed in the transient no-reflow group than in the reflow group. In addition, the frequency of the no-reflow phenomenon increased depending on the size of the lipid arc in the culprit plaque. ${ }^{44)}$ Distal protection has been proven to improve the reperfusion and to reduce the left ventricular dysfunction in patients with AMI who had angioscopically defined ruptured plaque. ${ }^{45}$ Thus, ruptured plaque, a large plaque burden, thin fibrous cap and thrombus formation are assumed to be predictors of the no-reflow phenomenon.

In the target lesion of PCI, positive remodeling and IVUS findings that may correspond to plaque rupture are associated with future cardiac events. ${ }^{46}$ ) On the hand, an- other investigator showed that culprit-lesion remodeling, rather than the presence or absence of culprit-lesion plaque rupture, was a strong predictor of the long-term clinical outcome in patients with ACS.47)

\section{Evaluation of medical treatment by intravascular imaging devices}

Several clinical trials have suggested that statin therapy could stabilize vulnerable plaques and reduce cardiovascular events. Intravascular imaging devices can show the effects of statins on the plaque morphology and components. An IVUS study showed early aggressive lipidlowering therapy using $20 \mathrm{mg}$ atorvastatin for 6 months significantly reduced the non-culprit plaque volume in patients with ACS. The percent change in plaque volume was correlated with the percent LDL-C reduction, even in patients with low baseline LDL-C. ${ }^{48}$ Another study demonstrated that statin therapy led to the regression of coronary atherosclerosis within 2 months following ACS.99) Furthermore, the administration of $4 \mathrm{mg}$ of pitavastatin was proven to be as effective to induce the regression of plaque volume as $20 \mathrm{mg}$ of atorvastatin. ${ }^{50}$ The thickness of coronary fibrous caps is a major determinant of plaque vulnerability. On OCT examination, the lipid-lowering therapy with statins for 9 months after the onset of AMI increased the fibrous-cap thickness. ${ }^{51)}$ These results provide insight into the rapid clinical benefits of statin therapy after ACS.

\section{Validation of noninvasive coronary artery imaging}

High performance computed tomography (CT) and magnetic resonance imaging (MRI) are useful for assessing coronary stenosis. Intravascular imaging devices play an important role in verifying the accuracy of plaque characterization by noninvasive coronary artery imaging. IVUS, study showed contrast-enhanced multidetector computed tomography (MDCT) more accurately assessed the plaque eccentricity, calcification, and remodeling, and the intraplaque hypodensity correlated with the echolucent area in patients with ACS. ${ }^{52,53)}$ MDCT identifies OCT-derived TCFA in the culprit lesions of ACS and SAP based on positive remodeling, low computed tomography attenuation values and ring-like enhancement. ${ }^{54}$ Due to the resolution of the techniques, small plaque structures cannot be assessed. The role of non-invasive imaging in vulnerable plaque detection is currently still being developed. 


\section{Other new technologies exploring intravascular imaging}

New intravascular devices have been developed to visualize more detailed characteristics of the coronary artery. Near-infrared spectroscopy (NIRS) reveals the chemical tissue characterizations based on the absorbance and scattering of near-infrared light. Thermography can detect plaque inflammation and neoangiogenesis by measuring the temperature of the plaque surface. Palpography, an IVUS-derived technique, measures the elasticity of the plaque. Three-dimensional IVUS assessment of the local shear stress is being developed as a practicable method to predict plaque rupture. These new intravascular devices will provide new insight into acute coronary syndrome.

\section{Conclusions}

Intravascular imaging devices have contributed to elucidate the pathology and to guide the clinical treatment of patients with ACS. In addition, intravascular imaging devices can be used to confirm the relevance of biomarkers and verify plaque characterization by noninvasive coronary artery imaging. The introduction of new technologies will further expand the potential of intravascular imaging devices for clinical application.

\section{References}

1) Nasu K, Tsuchikane E, Katoh O, Vince DG, Virmani R, Surmely JF, Murata A, Takeda Y, Ito T, Ehara M, Matsubara T, Terashima M, Suzuki T: Accuracy of in vivo coronary plaque morphology assessment: a validation study of in vivo virtual histology compared with in vitro histopathology. J Am Coll Cardiol 2006; 47: 2405-2412

2) Uchida Y, Uchida Y, Sakurai T, Kanai M, Shirai S, Morita T: Characterization of coronary fibrin thrombus in patients with acute coronary syndrome using dye-staining angioscopy. Arterioscler Thromb Vasc Biol 2011; 31: 1452-1460

3) Uchida Y, Uchida Y, Sakurai T, Kanai M, Shirai S, Oshima T, Koga A, Matsuyama A, Tabata T: Fluffy luminal surface of the non-stenotic culprit coronary artery in patients with acute coronary syndrome: an angioscopic study. Circ J 2010; 74: 2379-2385

4) Uchida Y, Uchida Y, Sakurai T, Kanai M, Shirai S, Oshima T, Koga A, Matsuyama A: Possible role of damaged neoendothelial cells in the genesis of coronary stent thrombus in chronic phase. A dye staining angioscopic study. Int Heart J 2011; 52: 12-16

5) Yabushita H, Bouma BE, Houser SL, Aretz HT, Jang IK, Schlendorf KH, Kauffman CR, Shishkov M, Kang DH,
Halpern EF, Tearney GJ: Characterization of human atherosclerosis by optical coherence tomography. Circulation 2002; 106: 1640-1645

6) Kume T, Akasaka T, Kawamoto T, Watanabe N, Toyota E, Neishi Y, Sukmawan R, Sadahira Y, Yoshida K: Assessment of coronary arterial plaque by optical coherence tomography. Am J Cardiol 2006; 97: 1172-1175

7) Takano M, Jang IK, Inami S, Yamamoto M, Murakami D, Okamatsu K, Seimiya K, Ohba T, Mizuno K: In vivo comparison of optical coherence tomography and angioscopy for the evaluation of coronary plaque characteristics. Am J Cardiol 2008; 101: 471-476

8) Kubo T, Imanishi T, Takarada S, Kuroi A, Ueno S, Yamano T, Tanimoto T, Matsuo Y, Masho T, Kitabata H, Tanaka A, Nakamura N, Mizukoshi M, Tomobuchi Y, Akasaka T: Implication of plaque color classification for assessing plaque vulnerability: a coronary angioscopy and optical coherence tomography investigation. JACC Cardiovasc Interv 2008; 1: 74-80

9) Kume T, Akasaka T, Kawamoto T, Ogasawara Y, Watanabe N, Toyota E, Neishi Y, Sukmawan R, Sadahira Y, Yoshida K: Assessment of coronary arterial thrombus by optical coherence tomography. Am J Cardiol 2006; 97:1713-1717

10) Tearney GJ, Yabushita H, Houser SL, Aretz HT, Jang IK, Schlendorf KH, Kauffman CR, Shishkov M, Halpern EF, Bouma BE: Quantification of macrophage content in atherosclerotic plaques by optical coherence tomography. Circulation 2003; 107: 113-119

11) Nakamura M, Nishikawa $H$, Mukai S, Setsuda M, Nakajima K, Tamada H, Suzuki H, Ohnishi T, Kakuta Y, Nakano T, Yeung AC: Impact of coronary artery remodeling on clinical presentation of coronary artery disease: an intravascular ultrasound study. J Am Coll Cardiol 2001; 37: 63-69

12) Hara H, Tsunoda T, Yamamoto M: The ultrasound attenuation behind coronary atheroma predicts embolic complication during percutaneous intervention. J Am Coll Cardiol 2005; 45(Suppl): 58A-59A

13) Hara H, Tsunoda T, Moroi M, Kubota T, Kunimasa T, Shiba M, Wada M, Tsuji T, Iijima R, Nakajima R, Yoshitama T, Nakamura M: Ultrasound attenuation behind coronary atheroma without calcification: mechanism revealed by autopsy. Acute Cardiac Care 2006; 8: 110-112

14) Ito $S$, Saio M, Suzuki T: Advanced atherosclerotic plaque as potential cause of no-reflow in elective percutaneous coronary intervention: intravascular ultrasound and histological findings. J Invasive Cardiol 2004; 16: 669-672

15) Hong MK, Mintz GS, Lee CW, Suh J, Kim JH, Park DW, Lee SW, Kim YH, Cheong SS, Kim JJ, Park SW, Park SJ: Comparison of virtual histology to intravascular ultrasound of culprit coronary lesions in acute coronary syndrome and target coronary lesions in stable angina pectoris. Am J Cardiol 2007; 100: 953-959

16) Wu X, Maehara A, Mintz GS, Kubo T, Xu K, Choi SY, He Y, Guo N, Moses JW, Leon MB, De Bruyne B, Serruys PW, Stone GW: Virtual histology intravascular ultrasound anal- 
ysis of non-culprit attenuated plaques detected by grayscale intravascular ultrasound in patients with acute coronary syndromes. Am J Cardiol 2010; 105: 48-53

17) MacNeill BD, Jang IK, Bouma BE, Iftimia N, Takano M, Yabushita H, Shishkov M, Kauffman CR, Houser SL, Aretz HT, DeJoseph D, Halpern EF, Tearney GJ: Focal and multifocal plaque macrophage distributions in patients with acute and stable presentations of coronary artery disease. J Am Coll Cardiol 2004; 44: 972-979

18) Ueda Y, Oyabu J, Okada K, Murakawa T, Hirayama A, Kodama K: Angioscopically-determined extent of coronary atherosclerosis is associated with severity of acute coronary syndrome. J Invasive Cardiol 2006; 18: 220-224

19) Mizuno K, Satomura K, Miyamoto A, Arakawa K, Shibuya T, Arai T, Kurita A, Nakamura H, Ambrose JA: Angioscopic evaluation of coronary-artery thrombi in acute coronary syndromes. N Engl J Med 1992; 326: 287-291

20) Ino Y, Kubo T, Tanaka A, Kuroi A, Tsujioka H, Ikejima H, Okouchi K, Kashiwagi M, Takarada S, Kitabata H, Tanimoto T, Komukai K, Ishibashi K, Kimura K, Hirata K, Mizukoshi M, Imanishi T, Akasaka T: Difference of culprit lesion morphologies between ST-segment elevation myocardial infarction and non-ST-segment elevation acute coronary syndrome: an optical coherence tomography study. JACC Cardiovasc Interv 2011; 4: 76-82

21) Hayashi T, Kiyoshima T, Matsuura M, Ueno M, Kobayashi N, Yabushita H, Kurooka A, Taniguchi M, Miyataka M, Kimura A, Ishikawa K: Plaque erosion in the culprit lesion is prone to develop a smaller myocardial infarction size compared with plaque rupture. Am Heart J 2005; 149: 284-290

22) Uchida Y, Nakamura F, Tomaru T, Morita T, Oshima T, Sasaki T, Morizuki S, Hirose J: Prediction of acute coronary syndromes by percutaneous coronary angioscopy in patients with stable angina. Am Heart J 1995; 130: 195-203

23) Ueda Y, Ohtani T, Shimizu M, Hirayama A, Kodama K: Assessment of plaque vulnerability by angioscopic classification of plaque color. Am Heart J 2004; 148: 333-335

24) Ishibashi F, Mizuno K, Kawamura A, Singh PP, Nesto RW, Waxman S: High yellow color intensity by angioscopy with quantitative colorimetry to identify high-risk features in culprit lesions of patients with acute coronary syndromes. Am J Cardiol 2007; 100: 1207-1211

25) Yamagishi M, Terashima M, Awano K, Kijima M, Nakatani S, Daikoku S, Ito K, Yasumura Y, Miyatake K: Morphology of vulnerable coronary plaque: insights from follow-up of patients examined by intravascular ultrasound before an acute coronary syndrome. J Am Coll Cardiol 2000; 35: 106-111

26) Stone GW, Maehara A, Lansky AJ, de Bruyne B, Cristea E, Mintz GS, Mehran R, McPherson J, Farhat N, Marso SP, Parise H, Templin B, White R, Zhang Z, Serruys PW; PROSPECT Investigators: A prospective natural-history study of coronary atherosclerosis. N Engl J Med 2011; 364: 226235

27) Surmely JF, Nasu K, Fujita H, Terashima M, Matsubara T,
Tsuchikane E, Ehara M, Kinoshita Y, Takeda Y, Tanaka N, Katoh O, Suzuki T: Association of coronary plaque composition and arterial remodelling: a virtual histology analysis by intravascular ultrasound. Heart 2007; 93: 928-932

28) Masumura Y, Ueda Y, Matsuo K, Akazawa Y, Nishio M, Hirata A, Kashiwase K, Nemoto T, Kashiyama T, Wada M, Muller JE, Kodama K: Frequency and location of yellow and disrupted coronary plaques in patients as detected by angioscopy. Circ J 2011; 75: 603-612

29) Rioufol G, Finet G, Ginon I, André-Fouët X, Rossi R, Vialle E, Desjoyaux E, Convert G, Huret JF, Tabib A: Multiple atherosclerotic plaque rupture in acute coronary syndrome: a three-vessel intravascular ultrasound study. Circulation 2002; 106: 804-808

30) Rodriguez-Granillo GA, McFadden EP, Valgimigli M, van Mieghem CA, Regar E, de Feyter PJ, Serruys PW: Coronary plaque composition of nonculprit lesions, assessed by in vivo intracoronary ultrasound radio frequency data analysis, is related to clinical presentation. Am Heart J 2006; 151: 1020-1024

31) Hong MK, Mintz GS, Lee CW, Lee JW, Park JH, Park DW, Lee SW, Kim YH, Cheong SS, Kim JJ, Park SW, Park SJ: A three-vessel virtual histology intravascular ultrasound analysis of frequency and distribution of thin-cap fibroatheromas in patients with acute coronary syndrome or stable angina pectoris. Am J Cardiol 2008; 101: 568-572

32) Rodriguez-Granillo GA, García-García HM, Mc Fadden EP, Valgimigli M, Aoki J, de Feyter P, Serruys PW: In vivo intravascular ultrasound-derived thin-cap fibroatheroma detection using ultrasound radiofrequency data analysis. J Am Coll Cardiol 2005; 46: 2038-2042

33) Kubo T, Imanishi T, Kashiwagi M, Ikejima H, Tsujioka H, Kuroi A, Ishibashi K, Komukai K, Tanimoto T, Ino Y, Kitabata H, Takarada S, Tanaka A, Mizukoshi M, Akasaka T: Multiple coronary lesion instability in patients with acute myocardial infarction as determined by optical coherence tomography. Am J Cardiol 2010; 105: 318-322

34) Kim SH, Hong MK, Park DW, Lee SW, Kim YH, Lee CW, Kim JJ, Park SW, Park SJ: Impact of plaque characteristics analyzed by intravascular ultrasound on long-term clinical outcomes. Am J Cardiol 2009; 103: 1221-1226

35) Ohtani T, Ueda Y, Mizote I, Oyabu J, Okada K, Hirayama A, Kodama K: Number of yellow plaques detected in a coronary artery is associated with future risk of acute coronary syndrome: detection of vulnerable patients by angioscopy. J Am Coll Cardiol 2006; 47: 2194-2200

36) Okamatsu K, Takano M, Sakai S, Ishibashi F, Uemura R, Takano T, Mizuno K: Elevated troponin T levels and lesion characteristics in non-ST-elevation acute coronary syndromes. Circulation 2004; 109: 465-470

37) Ohtani T, Ueda Y, Shimizu M, Mizote I, Hirayama A, Hori M, Kodama K: Association between cardiac troponin T elevation and angioscopic morphology of culprit lesion in patients with non-ST-segment elevation acute coronary syndrome. Am Heart J 2005; 150: 227-233 
38) Andrié RP, Bauriedel G, Braun P, Höpp HW, Nickenig G, Skowasch D: Increased expression of C-reactive protein and tissue factor in acute coronary syndrome lesions: Correlation with serum C-reactive protein, angioscopic findings, and modification by statins. Atherosclerosis 2009; 202: $135-143$

39) Tanaka A, Shimada K, Sano T, Namba M, Sakamoto T, Nishida Y, Kawarabayashi T, Fukuda D, Yoshikawa J: Multiple plaque rupture and C-reactive protein in acute myocardial infarction. J Am Coll Cardiol 2005; 45: 1594-1599

40) Otake H, Shite J, Shinke T, Watanabe S, Tanino Y, Ogasawara D, Sawada T, Hirata K, Yokoyama M: Relation between plasma adiponectin, high-sensitivity C-reactive protein, and coronary plaque components in patients with acute coronary syndrome. Am J Cardiol 2008; 101: 1-7

41) Li QX, Fu QQ, Shi SW, Wang YF, Xie JJ, Yu X, Cheng X, Liao YH: Relationship between plasma inflammatory markers and plaque fibrous cap thickness determined by intravascular optical coherence tomography. Heart 2010; 96: 196201

42) Fukuda D, Shimada K, Tanaka A, Kusuyama T, Yamashita H, Ehara S, Nakamura Y, Kawarabayashi T, Iida H, Yoshiyama M, Yoshikawa J: Comparison of levels of serum matrix metalloproteinase-9 in patients with acute myocardial infarction versus unstable angina pectoris versus stable angina pectoris. Am J Cardiol 2006; 97: 175-180

43) Iijima R, Shinji H, Ikeda N, Itaya H, Makino K, Funatsu A, Yokouchi I, Komatsu H, Ito N, Nuruki H, Nakajima R, Nakamura M: Comparison of coronary arterial finding by intravascular ultrasound in patients with "transient no-reflow" versus "reflow" during percutaneous coronary intervention in acute coronary syndrome. Am J Cardiol 2006; 97: 29-33

44) Tanaka A, Imanishi T, Kitabata H, Kubo T, Takarada S, Tanimoto T, Kuroi A, Tsujioka H, Ikejima H, Komukai K, Kataiwa H, Okouchi K, Kashiwaghi M, Ishibashi K, Matsumoto H, Takemoto K, Nakamura N, Hirata K, Mizukoshi M, Akasaka T: Lipid-rich plaque and myocardial perfusion after successful stenting in patients with non-STsegment elevation acute coronary syndrome: an optical coherence tomography study. Eur Heart J 2009; 30: 1348-1355

45) Mizote I, Ueda Y, Ohtani T, Shimizu M, Takeda Y, Oka T, Tsujimoto M, Hirayama A, Hori M, Kodama K: Distal protection improved reperfusion and reduced left ventricular dysfunction in patients with acute myocardial infarction who had angioscopically defined ruptured plaque. Circulation 2005; 112: 1001-1007

46) Okura H, Taguchi H, Kubo T, Toda I, Yoshiyama M, Yoshikawa J, Yoshida K: Impact of arterial remodelling and plaque rupture on target and non-target lesion revascularisation after stent implantation in patients with acute coronary syndrome: an intravascular ultrasound study. Heart 2007; 93: 1219-1225
47) Okura H, Kobayashi Y, Sumitsuji S, Terashima M, Kataoka T, Masutani M, Ohyanagi M, Shimada K, Taguchi H, Yasuga Y, Takeda Y, Ohashi Y, Awano K, Fujii K, Mintz GS: Effect of culprit-lesion remodeling versus plaque rupture on three-year outcome in patients with acute coronary syndrome. Am J Cardiol 2009; 103: 791-795

48) Okazaki S, Yokoyama T, Miyauchi K, Shimada K, Kurata T, Sato H, Daida H: Early statin treatment in patients with acute coronary syndrome: demonstration of the beneficial effect on atherosclerotic lesions by serial volumetric intravascular ultrasound analysis during half a year after coronary event: the ESTABLISH Study. Circulation 2004; 110: 1061-1068

49) Rodés-Cabau J, Tardif JC, Cossette M, Bertrand OF, Ibrahim R, Larose E, Grégoire J, L' allier PL, Guertin MC: Acute effects of statin therapy on coronary atherosclerosis following an acute coronary syndrome. Am J Cardiol 2009; 104: 750-757

50) Hiro T, Kimura T, Morimoto T, Miyauchi K, Nakagawa $\mathrm{Y}$, Yamagishi M, Ozaki Y, Kimura K, Saito S, Yamaguchi T, Daida H, Matsuzaki M; JAPAN-ACS Investigators: Effect of intensive statin therapy on regression of coronary atherosclerosis in patients with acute coronary syndrome: a multicenter randomized trial evaluated by volumetric intravascular ultrasound using pitavastatin versus atorvastatin (JAPAN-ACS [Japan assessment of pitavastatin and atorvastatin in acute coronary syndrome] study). J Am Coll Cardiol 2009; 54: 293-302

51) Takarada S, Imanishi T, Kubo T, Tanimoto T, Kitabata H, Nakamura N, Tanaka A, Mizukoshi M, Akasaka T: Effect of statin therapy on coronary fibrous-cap thickness in patients with acute coronary syndrome: assessment by optical coherence tomography study. Atherosclerosis 2009; 202: 491-497

52) Caussin C, Ohanessian A, Ghostine S, Jacq L, Lancelin B, Dambrin G, Sigal-Cinqualbre A, Angel CY, Paul JF: Characterization of vulnerable nonstenotic plaque with 16 -slice computed tomography compared with intravascular ultrasound. Am J Cardiol 2004; 94: 99-104

53) Hoffmann U, Moselewski F, Nieman K, Jang IK, Ferencik M, Rahman AM, Cury RC, Abbara S, Joneidi-Jafari H, Achenbach S, Brady TJ: Noninvasive assessment of plaque morphology and composition in culprit and stable lesions in acute coronary syndrome and stable lesions in stable angina by multidetector computed tomography. J Am Coll Cardiol 2006; 47: 1655-1662

54) Kashiwagi M, Tanaka A, Kitabata H, Tsujioka H, Kataiwa H, Komukai K, Tanimoto T, Takemoto K, Takarada S, Kubo T, Hirata K, Nakamura N, Mizukoshi M, Imanishi T, Akasaka T: Feasibility of noninvasive assessment of thin-cap fibroatheroma by multidetector computed tomography. JACC Cardiovasc Imaging 2009; 2: 1412-1419 